\title{
Augmenting Community Narratives: An Exploration of Lens-based Image and Publication Design
}

\author{
Keywords \\ Visual Literacy; Media; Technologies of the Image; Design Education; Design Studio.
}

This presentation proposal advances a pedagogical case study that focuses on innovative ways of promoting visual literacy for undergraduate students through lens-based image-making and digital technologies. The curriculum was designed to be delivered throughout an academic semester at Auckland University of Technology South Campus, Manukau City, Aotearoa New Zealand. The content is formatted for first-year students of the Diploma in Arts endorsed in Communication Design. The Diploma in Arts is appealing to students who may be seeking a shorter design qualification. In addition, the programme provides for students who have not met or have narrowly missed University Entrance (UE) criteria. The presentation focuses on the structure and contents of the pedagogical experience where lecturers and students are connected in the design studio through agency and literacy rather than just observing pedagogical prescriptions. In this active environment, the hidden curriculum that deals with the participant's cultural background and natural epistemologies is valued and validated. In creating the engagement for visual literacy, the brief requires students to consider aspects of community, taking the role of a storyteller equipped with skills to create visual content that is meaningful and actual. The course is divided into two parallel design studio approaches: one that deals with visual literacy through the photographic image and practice; and the other is concerned with aspects of the nature of the media, augmented reality and image dissemination in digital platforms. The first area focused on visual literacy through photographic practice, using the politics of photographic language and representation, employing portraiture analysis and image creation. This approach encompasses the conceptual aspects associated with photographic images and the technical aspects of lens-based image-making. The second area focused on understanding digital media, the application of tools and how to take advantage of the interactive environment to promote awareness and reflect change in the community. This part of the brief intended to create an understanding of the issues associated with online environments, providing students with skills to operate creatively and fluidly in response to the fast-paced nature of online image-sharing platforms. Within this context, techniques of digital image construction and manipulation were investigated in the light of industry applications and best practice workflows. These learning areas were underpinned by an online blog where students actively recorded their design process, tests, and contextual influences that impacted their design practice. The studio environment fostered an inclusive and participatory form of teaching and learning and employed students' smartphones as an accessible tool to produce and understand images. The project contributes to knowledge about the design studio through a framework for Visual literacy and media education, where students learn principles of photography practice and digital technologies. It contributes to reflections about the use of mobile phone technologies as a common entry way apparatus to visual literacy and imagery generator. 